lems involved in quantifying the impact of man on the global cycles.

The terrestrial bias may be excused in part because the immediate impact of man is most apparent on land. However, by inadvertently relegating the oceans to a secondary role, many of the feedbacks that are crucial to the stability of the biospheric cycles over the medium term are not considered. Indeed, the book deals unconvincingly with the overall potential for feedback in the natural cycles despite Smil's recognition of the "endless opportunities for interaction within and among individual cycles". The insights and provocative analyses of Lovelock in relation to feedback and regulation in the biosphere receive no mention, even though the early contributions of Vernadsky are recognized.

The book closes with an enigmatic quotation from Vernadsky in which he suggests that we are "in a transition to the noosphere", a concept related by Smil, in another quotation from Vernadsky, to "the reconstruction of the biosphere (my italics) in the interests of freely thinking humanity as a single totality". After reading this book I recoil from this vision! We should rather understand our dependence on these grand cycles, and learn to tread softly in their presence.

Mike Whitfield is Director of the Marine Biological Association of the United Kingdom, The Laboratory, Citadel Hill, Plymouth PL1 2PB, UK.

\section{Microbes writ large}

\section{Life at Small Scale: The Behavior of Microbes}

by David B. Dusenbery

Scientific American Library/W. H. Freeman: 1996. Pp. 214. \$32.95, £19.95

\section{Roger Whittenbury}

"A word means what I want it to mean" would (with apologies to Lewis Carroll) be an apt subtitle for this book. Imprecise as the term 'microbe' is, most microbiologists indeed biologists - would agree that at the very least a microscope rather than a magnifying glass is required to see such a creature. The author does not subscribe to this view. Idiosyncratically, he opts for a functional definition: that microbes are organisms small enough to prosper without a circulatory system. This novel definition allows him to include in his 'microbial net' beasts such as nematodes and flatworms, and is an early indication of his unconventional approach. It is, in fact, hard to say exactly what message he is seeking to convey. The book comes across largely as a compilation of the author's interests, with readers left to get from it whatever they can.

The main themes explored are how microbes move, navigate and communicate, and there are diversions into survival and

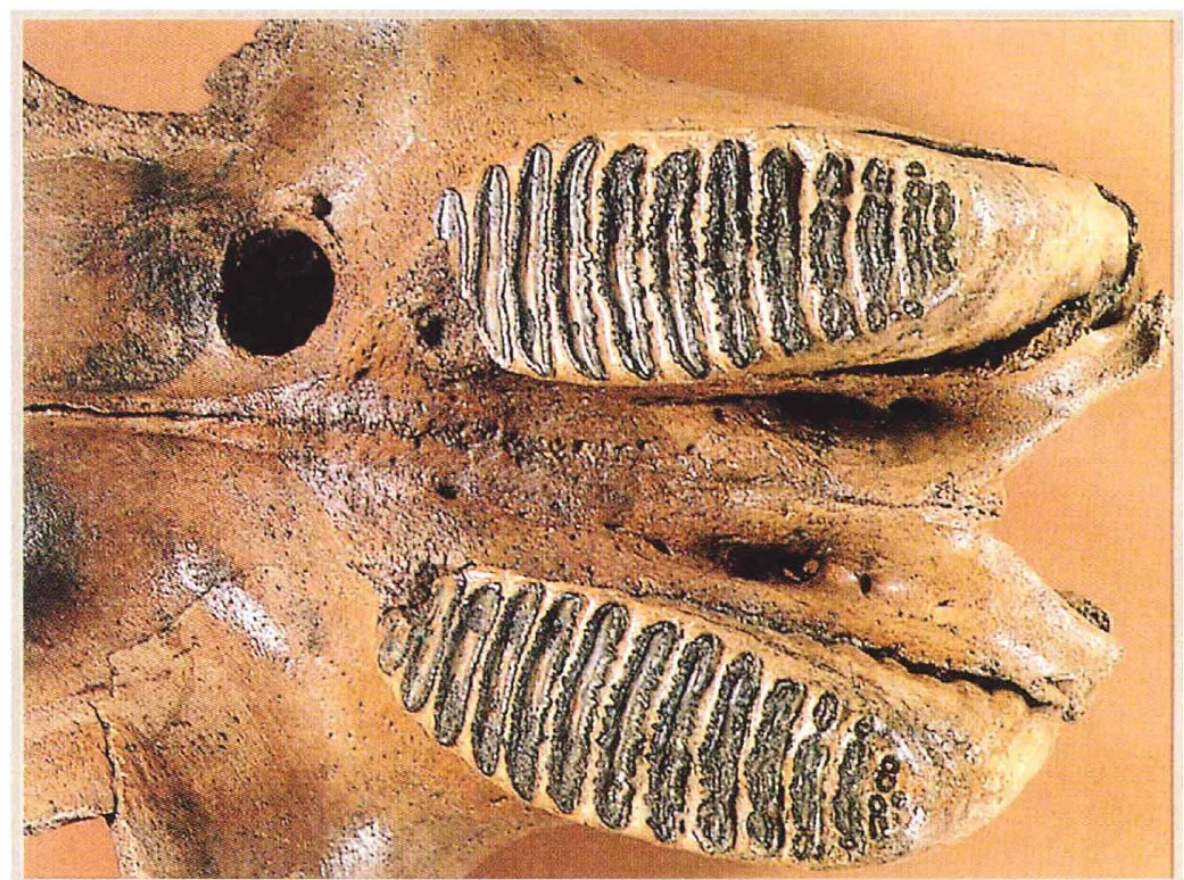

\title{
Even elephants get toothache
}

This fossilized jaw of Phalaeoloxodon, an elephant from the Pleistocene epoch, has a circular hole by the left molar caused by

feeding strategies. The result is a curious but interesting mix of detailed analyses, often with biophysical overtones (reflecting the author's background), basic commentary, and speculation. All this is accompanied by superb colour photographs and clear diagrams.

Ideally, readers should have at their side a standard text on microbiology, because the author makes generalizations that sometimes are not as all-embracing or accurate as they might have been. For instance, not all photosynthetic organisms are aerobic photoautotrophs that synthesize macromolecules from inorganic nutrients, carbon dioxide and water, releasing oxygen as a byproduct. Many can photosynthesize only in the absence of oxygen, using a range of inorganic and organic electron donors other than water.

Occasional contradictory statements and apparent ambivalence on the author's part may be off-putting for the nonspecialist reader. The author says that Synechococcus, a photosynthetic cyanobacterium, produces oxygen in the dark and fixes nitrogen in the light, but on the next page we are told (correctly) the opposite. He also states that bacteria are too small to detect gradients of nutrients, suggesting that they have no choice but to settle for the niche in which they find themselves. Yet later on there is an extensive and excellent essay on chemotaxis, the process whereby motile bacteria can move towards or away from substances - in effect, up and down gradients. Although sensing a gradient along its surface might not be a property of a particular bacterium, the tooth decay. Helmut Mayr's A Guide to Fossils covers a wide range of fossils, and is out now in paperback (Princeton University Press, \$18.95).

author's treatment will undoubtedly puzzle some readers.

The ability of photosynthetic organisms to coexist despite their different physiological requirements is a fascinating story of evolutionary adaptation, tailor-made for a text of this type. Sadly, by presenting the facts too briefly and too simply, the author misses this opportunity. The description of chemoautotrophs provides another example of the dangers of oversimplification. The author gives the impression that all such organisms are strict anaerobes. In fact, most are strict aerobes that play an essential part in global nutrient recycling, using oxygen as an electron acceptor in the oxidation of a wide range of inorganic compounds such as ammonia, nitrite and ferrous sulphide.

The chapter on "Locomotion without legs" is a tour de force, however, heavily underpinned by explanations of the way in which physical principles dictate movement in water and how small organisms have adapted to viscosity and other physical constraints. Other chapters are more variable in content and detail, and less focused.

Overall, this is an interesting but not unflawed work, filling no particular niche. The intelligent layperson will find many parts difficult to comprehend but will be charmed by others. The author clearly is not troubled by such issues. He dedicates the book "To all who share in the wonder of life on Earth"!

Roger Whittenbury is in the Department of Biological Sciences, University of Warwick, Coventry CV4 7AL, UK. 